\title{
Protection against Plasmodium falciparum malaria by PfSPZ Vaccine
}

Judith E. Epstein, ${ }^{1}$ Kristopher M. Paolino, ${ }^{2}$ Thomas L. Richie, ${ }^{3}$ Martha Sedegah, ${ }^{1}$ Alexandra Singer, ${ }^{1}$ Adam J. Ruben, ${ }^{3}$ Sumana Chakravarty, ${ }^{3}$ April Stafford, ${ }^{2}$ Richard C. Ruck, ${ }^{2}$ Abraham G. Eappen, ${ }^{3}$ Tao Li, ${ }^{3}$ Peter F. Billingsley, ${ }^{3}$ Anita Manoj, ${ }^{3}$ Joana C. Silva, ${ }^{4}$ Kara Moser, ${ }^{4}$ Robin Nielsen, ${ }^{2}$ Donna Tosh, ${ }^{2}$ Susan Cicatelli, ${ }^{2}$ Harini Ganeshan, ${ }^{1}$ Jessica Case, ${ }^{5}$ Debbie Padilla, ${ }^{3}$ Silas Davidson, ${ }^{2}$ Lindsey Garver, ${ }^{2}$ Elizabeth Saverino, ${ }^{3}$ Tooba Murshedkar, ${ }^{3}$ Anusha Gunasekera, ${ }^{3}$ Patrick S. Twomey, ${ }^{2}$ Sharina Reyes, ${ }^{1}$ James E. Moon, ${ }^{2}$ Eric R. James, ${ }^{3}$ Natasha KC, ${ }^{3,6}$ Minglin Li, ${ }^{3,6}$ Esteban Abot, ${ }^{1}$ Arnel Belmonte, ${ }^{1}$ Kevin Hauns, ${ }^{2}$ Maria Belmonte, ${ }^{1}$ Jun Huang, ${ }^{1}$ Carlos Vasquez, ${ }^{1}$ Shon Remich, ${ }^{2}$ Mary Carrington, ${ }^{1}$ Yonas Abebe, ${ }^{3}$ Amy Tillman, ${ }^{5}$ Bradley Hickey, ${ }^{2}$ Jason Regules, ${ }^{2}$ Eileen Villasante, ${ }^{1}$ B. Kim Lee Sim, ${ }^{3,6}$ and Stephen L. Hoffman ${ }^{3}$

${ }^{1}$ Naval Medical Research Center (NMRC), "'Walter Reed Army Institute of Research (WRAIR), Silver Spring, Maryland, USA. ${ }^{3}$ Sanaria Inc., Rockville, Maryland, USA. Institute for Genome Sciences, University of Maryland School of Medicine, Baltimore, Maryland, USA. ${ }^{5}$ Statistics Collaborative Inc., Washington, DC, USA. ${ }^{6}$ Protein Potential, Rockville, Maryland, USA.

BACKGROUND: A radiation-attenuated Plasmodium falciparum (Pf) sporozoite (SPZ) malaria vaccine, PfSPZ Vaccine, protected 6 of 6 subjects (100\%) against homologous Pf (same strain as in the vaccine) controlled human malaria infection (CHMI) 3 weeks after 5 doses administered intravenously. The next step was to assess protective efficacy against heterologous Pf (different from Pf in the vaccine), after fewer doses, and at 24 weeks.

METHODS: The trial assessed tolerability, safety, immunogenicity, and protective efficacy of direct venous inoculation (DVI) of 3 or $\mathbf{5}$ doses of PfSPZ Vaccine in non-immune subjects.

RESULTS: Three weeks after final immunization, 5 doses of $2.7 \times 10^{5} \mathrm{PfSPZ}$ protected 12 of 13 recipients (92.3\% [95\% Cl: 48.0, 99.8]) against homologous CHMI and 4 of 5 (80.0\% [10.4, 99.5]) against heterologous CHMI; 3 doses of $4.5 \times 10^{5}$ PfSPZ protected 13 of 15 (86.7\% [35.9, 98.3]) against homologous CHMI. Twenty-four weeks after final immunization, the 5-dose regimen protected 7 of $10(70.0 \%[17.3,93.3])$ against homologous and 1 of $10(10.0 \%[-35.8,45.6])$ against heterologous $\mathrm{CHMI}$; the 3 -dose regimen protected 8 of $14(57.1 \%[21.5,76.6])$ against homologous CHMI. All 22 controls developed Pf parasitemia. PfSPZ Vaccine was well tolerated, safe, and easy to administer. No antibody or T cell responses correlated with protection.

CONCLUSIONS: We have demonstrated for the first time to our knowledge that PfSPZ Vaccine can protect against a 3-week heterologous CHMI in a limited group of malaria-naive adult subjects. A 3-dose regimen protected against both 3-week and 24-week homologous CHMI ( $87 \%$ and $57 \%$, respectively) in this population. These results provide a foundation for developing an optimized immunization regimen for preventing malaria.

TRIAL REGISTRATION: ClinicalTrials.gov NCT02215707.

FUNDING: Support was provided through the US Army Medical Research and Development Command, Military Infectious Diseases Research Program, and the Naval Medical Research Center's Advanced Medical Development Program.

sponsor of the clinical trial. In addition,

S.L. Hoffman and B.K.L. Sim have a

financial interest in Sanaria Inc.

Submitted: June 22, 2016

\section{Introduction}

In 2014 approximately $\$ 2.5$ billion was invested in malaria control, and there were at least 214 million clinical cases and 438,000 deaths caused by malaria, with infants and children in Africa bearing the greatest burden (1). Malaria is also a threat to travelers; the US Department of Defense has ranked malaria as its number one infectious threat. A highly effective malaria vaccine would be an ideal tool to 
Table 1. Demographics of study subjects

\begin{tabular}{|c|c|c|c|c|}
\hline & Groups 1 and 2 & Group 3 & Infectivity controls & Total \\
\hline Subjects enrolled, $n$ & 30 & 15 & 22 & 67 \\
\hline Age, yr; mean (SD) & $30.2(6.6)$ & $30.8(7.1)$ & $28.1(7.5)$ & $29.6(7.0)$ \\
\hline Range (min, max) & 19, 41 & 22,43 & 19,45 & 19,45 \\
\hline Male, $n(\%)$ & $16(53)$ & $7(47)$ & $13(59)$ & $36(54)$ \\
\hline Female, $n(\%)$ & $14(47)$ & $8(53)$ & $9(41)$ & $31(46)$ \\
\hline \multicolumn{5}{|l|}{ Race, $n(\%)$} \\
\hline White & $14(47)$ & $8(53)$ & $13(59)$ & $35(52)$ \\
\hline Black or African American & $14(47)$ & $7(47)$ & $9(41)$ & $30(45)$ \\
\hline Asian & $1(3)$ & 0 & 0 & $1(1)$ \\
\hline $\begin{array}{l}\text { Native Hawaiian or Other } \\
\text { Pacific Islander }\end{array}$ & $1(3)$ & 0 & 0 & $1(1)$ \\
\hline \multicolumn{5}{|l|}{ Ethnicity, n (\%) } \\
\hline Not Hispanic or Latino & $27(90)$ & $14(93)$ & $17(77)$ & $58(87)$ \\
\hline Hispanic or Latino & $3(10)$ & $1(7)$ & $5(23)$ & $9(13)$ \\
\hline Military member, $n$ (\%) & $10(33)$ & $7(47)$ & $5(23)$ & $22(33)$ \\
\hline \multicolumn{5}{|l|}{$\mathrm{BMI}\left(\mathrm{kg} / \mathrm{m}^{2}\right)$} \\
\hline Mean (SD) & $27.6(4.8)$ & $25.9(4.0)$ & $26.9(4.6)$ & $27.0(4.5)$ \\
\hline Range (min, $\max$ ) & 20,40 & 19, 33 & 19, 33 & 19,40 \\
\hline
\end{tabular}

Number of subjects enrolled (top row) was used as a denominator for all percentages. prevent malaria in travelers and deployed military, reduce morbidity and mortality in infants and children, and eliminate malaria from defined geographic areas through mass vaccine administration campaigns.

Plasmodium falciparum (Pf) sporozoites (SPZ) are the only immunogens ever shown to induce $>90 \%$, sterile protective immunity against malaria (2-4). Until recently, this had only been accomplished by immunization by mosquito bite. Manufacturing processes have now been developed for the production of aseptic, purified, vialed PfSPZ Vaccine (5). In the first clinical trial Sanaria PfSPZ Vaccine administered subcutaneously or intradermally was well tolerated and safe, but poorly protective (6). Studies in non-human primates indicated the vaccine would be protective if administered by intravenous (i.v.) injection (6). In the second clinical trial, 5 doses of PfSPZ Vaccine administered by i.v. injection protected 6 of $6(100 \%)$ subjects against con-

trolled human malaria infection (CHMI) with homologous (same as in vaccine) Pf parasites 3 weeks after final immunization (4). In a third trial, 55\% protection was achieved at 14 months after a 4-dose immunization regimen (7).

The WHO Malaria Vaccine Technology Roadmap (2013 update) set a goal for the year 2030 of a malaria vaccine with protective efficacy of at least $75 \%$ against clinical malaria, and development of malaria vaccines that reduce transmission of the parasite (8). We have focused on establishment of a regimen that would protect at least $80 \%$ of individuals against all Pf infections and could be administered with a maximum of 3 doses. Such a vaccine would meet all WHO objectives. To address these objectives, and to assess the efficiency and tolerability of administering the vaccine by direct venous inoculation (DVI), we designed a study in which numbers of PfSPZ/dose, numbers of doses, and intervals between doses were altered. Protection was assessed at 3 and 24 weeks after the last immunization against homologous Pf parasite challenge and, for the first time to our knowledge, heterologous (different genetically from parasites in vaccine) Pf parasite challenge. Furthermore, we assessed the efficiency and tolerability of DVI administration of PfSPZ.

\section{Results}

\section{Genetic differences between $P$. falciparum isolates}

Pf7G8 had been used in the 1990s to establish heterologous protection in volunteers immunized by the bite of irradiated mosquitoes carrying Pf sporozoites (9). To establish that Pf7G8 was representative of a heterologous strain, we utilized whole genome sequencing data to estimate genetic distance between the vaccine strain, PfNF54, and Pf7G8, and between these and 19 clinical isolates from Africa (Supplemental Table 1 and Supplemental Figure 1; supplemental material available online with this article; http://doi.org/10.1172/jci.insight.89154DS1). Comparison of 3D7 (a proxy for its parent PfN54) and 7G8 revealed 22,056 SNPs genome-wide, an average of 0.95 SNPs/ kb. 4,925 SNPs fall within a panel of $\sim 10^{6}$ validated, protein-coding SNPs. This number of SNPs relative to $3 \mathrm{D} 7$ is comparable to that from African Pf clinical isolates (Supplemental Figure 1). For 13 selected pre-erythrocytic Pf antigens and vaccine candidates, non-synonomous SNPs in 7G8 relative to NF54 were common in all genes, including 8 in the PfCSP gene (Supplemental Table 2), confirming the genetic differentiation between the two strains. 


\section{Study population}

Forty-five immunized and 24 infectivity control subjects were enrolled. Subjects were recruited from the Baltimore-Washington, DC, area. Baseline demographics were balanced. Study volunteer demographics and baseline characteristics are presented by group for all enrolled subjects (Table 1). Both civilian and military personnel were included. Ten of the 30 subjects in groups 1 and $2(33 \%)$ and 7 of the 15 subjects in group 3 (47\%) were military members.

Forty-one of 45 subjects (91\%) completed all scheduled vaccinations. In the $4.5 \times 10^{5} \mathrm{PfSPZ}$ group (group 3), all 15 subjects received all immunizations and proceeded to CHMI \#1. In the $2.7 \times 10^{5} \mathrm{PfSPZ}$ group (groups 1 and 2), 26 of 30 subjects received all 5 doses (were "fully immunized") and were available for CHMI. Two additional subjects in the $2.7 \times 10^{5} \mathrm{PfSPZ}$ group who did not receive all 5 scheduled immunizations proceeded to CHMI. Of the 28 subjects immunized in the $2.7 \times 10^{5} \mathrm{PfSPZ}$ groups who were available for CHMI, 14 were randomized to group 1 (homologous CHMI) and 14 were randomized to group 2 (heterologous CHMI). Two subjects randomized to heterologous CHMI did not receive CHMI. Of the two remaining subjects who received $2.7 \times 10^{5} \mathrm{PfSPZ}$ immunizations, one subject missed the last immunization and both CHMIs due to deployment overseas, and one subject discontinued from the study after receiving 4 doses (Figure 1).

Randomization was performed using a random generator program (simple randomization). Subjects were informed by a study investigator of their CHMI group approximately 3 weeks prior to first CHMI. Infectivity controls, who did not receive vaccine or placebo, were not recruited and enrolled until the time of each CHMI; thus they were not randomized.

CHMI \#1 at 3 weeks after last vaccine dose. Fourteen subjects in group 1 (13 of whom who were fully immunized) and 15 subjects in group 3 (all fully immunized) underwent Pf3D7 CHMI. There were 6 Pf3D7 CHMI infectivity controls. A total of 5 fully immunized subjects in group 2 underwent Pf7G8 CHMI. There were 4 Pf7G8 CHMI infectivity controls. One of the 5 vaccinees in the analysis population was bitten by only 3 Pf7G8-infected mosquitoes, and 1 of the 4 infectivity controls was bitten by only 4 Pf7G8-infected mosquitoes. Six of 11 fully immunized subjects in group 2 and 2 of 6 infectivity controls did not undergo Pf7G8 CHMI due to poor mosquito feeding.

CHMI \#2 at 24 weeks after last dose of vaccine. Eleven subjects in group 1 (10 of whom who were fully immunized), 14 subjects in group 3 (all fully immunized), and 6 infectivity controls underwent Pf3D7 CHMI. Twelve subjects in group 2 (11 of whom who were fully immunized) and 6 infectivity controls underwent Pf7G8 CHMI. One subject in group 2 was dropped from the analysis population because of non-compliance.

\section{Protective efficacy}

CHMI \#1. Participants underwent CHMI \#1 3 weeks after treatment with the final dose of vaccine (Figure 2, Table 2, Supplemental Table 3, and Supplemental Table 4).

Group 1: Subjects received 5 doses of $2.7 \times 10^{5}$ PfSPZ (homologous Pf3D7 CHMI). All 6 controls developed parasitemia with a median prepatent period of 11.6 days. Twelve of 13 fully immunized subjects did not develop parasitemia, providing a protective efficacy of $92.3 \%$ (95\% confidence interval [CI]: 48.0, 99.8, $P=0.0003$ ). The prepatent period in the one immunized subject who developed parasitemia was 13.9 days.

Group 2: Subjects received 5 doses of $2.7 \times 10^{5} \mathrm{PfSPZ}$ (heterologous Pf7G8 CHMI). All 4 controls exposed to the bites of $5(n=3)$ or $4(n=1)$ PfSPZ-infected mosquitoes developed parasitemia, with a median prepatent period of 11.9 days. Four of 5 fully immunized subjects exposed to the bite of $5(n=4)$ or $3(n=1)$ infected mosquitoes did not develop parasitemia. The protective efficacy of this cohort was $80.0 \%$ (95\% CI: 10.4 99.5, $P=0.048$ ). The prepatent period in the one fully immunized subject who developed parasitemia was 11.9 days. A fully immunized subject who was exposed to only 3 PfSPZ-infected mosquitoes was one of the 4 subjects counted as protected. One fully immunized subject was excluded from the analysis population due to non-compliance.

Group 3: Subjects received 3 doses of $4.5 \times 10^{5} \mathrm{PfSPZ}$ (homologous Pf3D7 CHMI). Controls were the same as those in group 1 . Thirteen of 15 fully immunized subjects did not develop parasitemia, providing a protective efficacy of $86.7 \%$ (95\% CI: 35.9, 98.3, $P=0.0005)$. The prepatent periods in the 2 immunized subjects who developed parasitemia were 13.9 and 16.9 days.

CHMI \#2. Participants underwent CHMI \#2 24 weeks after treatment with the final dose of vaccine (Figure 3, Table 2, Supplemental Table 3, and Supplemental Table 4). 


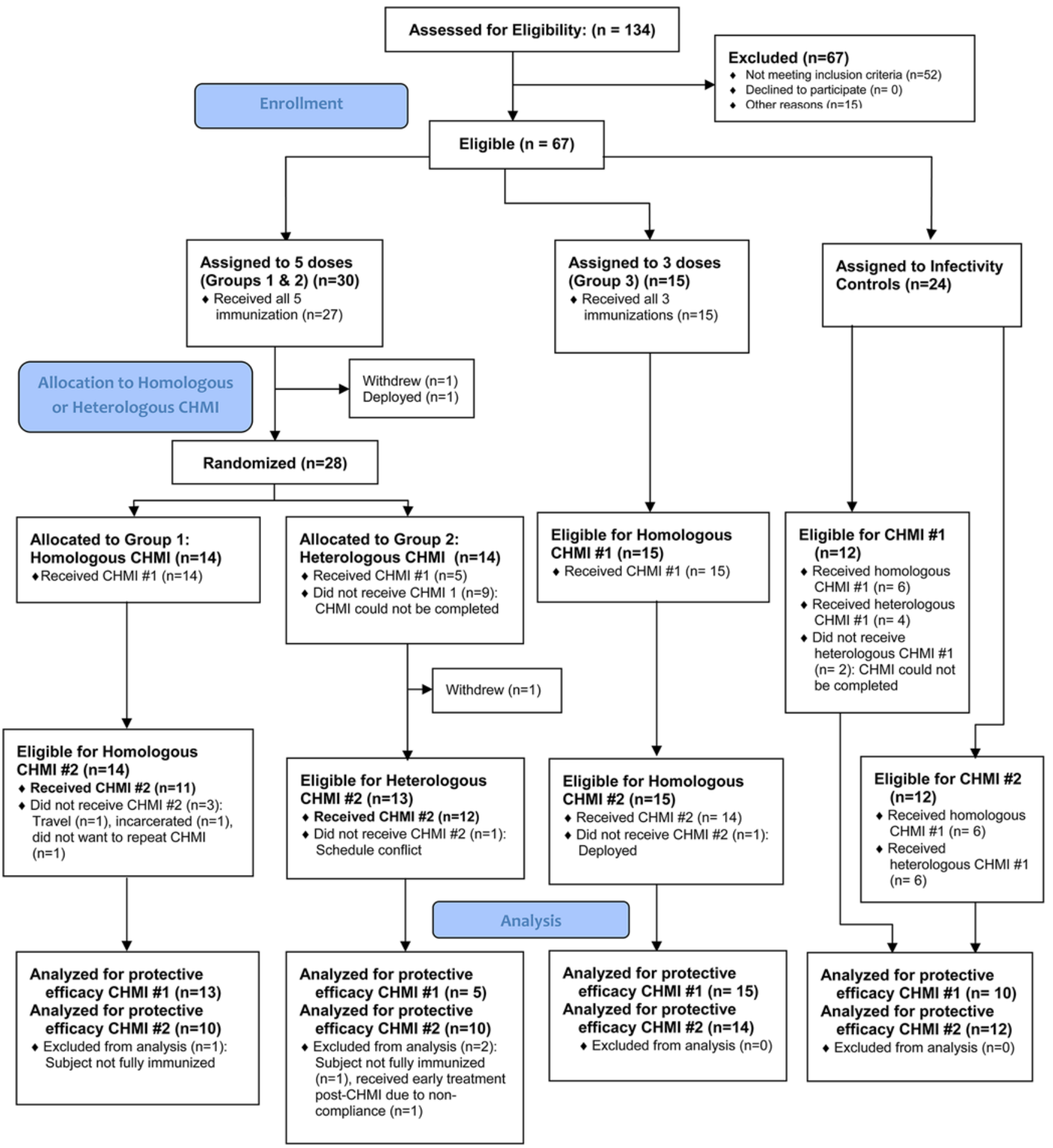

Figure 1. Consort 2010 flow diagram. Thirty subjects were enrolled to receive 5 doses of $2.7 \times 10^{5} \mathrm{PfSPZ}$ /dose at weeks 0 , 4, 8, 12, and 20; subjects were then randomized equally to group 1 (homologous CHMIs) or group 2 (heterologous CHMIs). Fifteen subjects were enrolled to receive 3 doses of $4.5 \times 10^{5}$ $\mathrm{PfSPZ}$ /dose at weeks 0, 8, and 16 with homologous CHMIs (group 3, which began immunizations 1 month after groups 1 and 2). Twenty-four subjects were enrolled as infectivity controls for the total of 4 CHMIs (2 homologous CHMIs and 2 heterologous CHMIs); each CHMI included 6 infectivity controls, except heterologous CHMI \#1, for which only 4 infectivity controls were challenged. CHMI, controlled human malaria infection. 
A
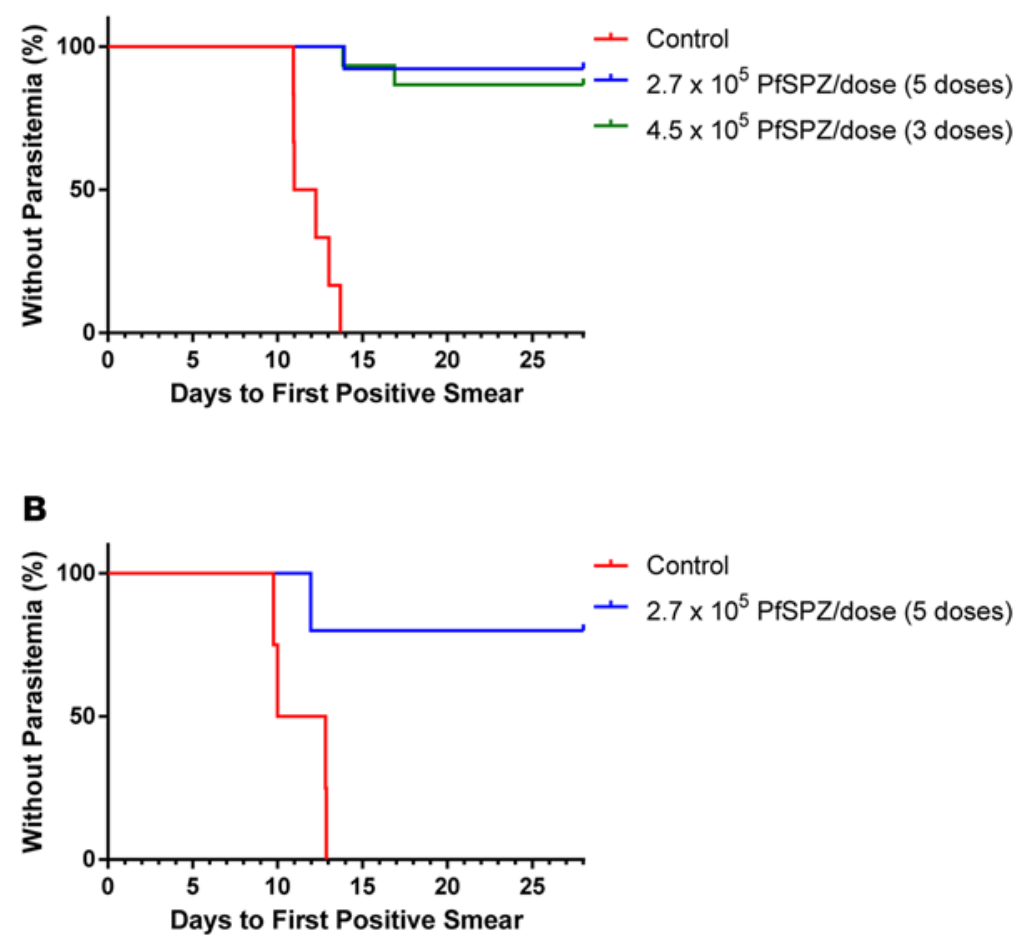

Figure 2. Results of CHMI done 3 weeks after the last dose of PfSPZ Vaccine. (A and B) Survival curves in volunteers undergoing CHMI 3 weeks after the last of 5 doses of $2.7 \times 10^{5} \mathrm{PfSPZ}$ (A and $\mathbf{B}$ ) or 3 doses of $4.5 \times 10^{5} \mathrm{PfSPZ}$ (A). Homologous $\mathrm{CHMI}$ with Pf $3 D 7$ parasites was analyzed in two immunized groups (group $1\left[2.7 \times 10^{5} \mathrm{PfSPZ}\right]: n=13$; group $3\left[4.5 \times 10^{5} \mathrm{PfSPZ}\right]: n=15$ ) and one control group $(n=6)(\mathbf{A})$. Heterologous CHMI with Pf 7C8 parasites was analyzed in one immunized group (group 2 [2.7 $\times$ $\left.\left.10^{5} \mathrm{PfSPZ}\right]: n=5\right)$ and one control group $(n=4)(B)$. CHMI, controlled human malaria infection; Pf, Plasmodium falciparum.

Group 1: All 6 controls developed parasitemia, with a median prepatent period of 11.6 days. Seven of 10 fully immunized subjects who underwent CHMI \#2 did not develop parasitemia, providing a protective efficacy of $70 \%$ (95\% CI: $17.3,93.3, P=0.011)$. The median prepatent period in the 3 fully immunized subjects who developed parasitemia was 15.4 days.

Group 2: All 6 controls developed parasitemia, with a median prepatent period of 10.9 days. One of 10 fully immunized subjects did not develop parasitemia, providing a protective efficacy of $10.0 \%$ (95\% CI: $-35.8,45.6$, $P=1.00)$. The subject who did not develop parasitemia was the subject who did not develop parasitemia in the first CHMI when exposed to the bites of 3 PfSPZ-infect-

ed mosquitoes. The median prepatent period in the 9 fully immunized subjects who developed parasitemia was 11.9 days. When comparing the prepatent period in the group 2 subjects who developed parasitemia with the controls, there was a statistically significant delay of 1 day (Wilcoxon rank-sum exact test 2-sided $P$ $=0.005$ ). One fully immunized subject was excluded from the analysis population due to non-compliance.

Group 3: Controls were the same as those in group 1. Eight of 14 fully immunized subjects did not develop parasitemia, providing a protective efficacy of $57.1 \%(95 \% \mathrm{CI}: 21.5,76.6, P=0.042)$. The median prepatent period in the 6 immunized subjects who developed parasitemia was 14.0 days.

Results for the challenged population, including subjects who missed one or more immunizations, are presented in Supplemental Table 3. All protected individuals were negative for Pf parasites by real-time quantitative PCR (qPCR) on their visits 11, 18, and 28 days after CHMI. All individuals positive by thick blood smear (TBS) were positive by qPCR.

\section{Antibody responses}

PfCSP and PfSPZ. Antibodies against PfCSP by ELISA, PfSPZ by automated immunofluorescence assay (aIFA), and PfSPZ by inhibition of sporozoite invasion (ISI) assay in sera taken 2 weeks after the last dose of vaccine and just prior to the second CHMI ( $\sim 24$ weeks after last dose of vaccine) for the 3 groups are shown in Figure 4, A-C. The median response (bar with number) and those protected (filled symbols) and not protected (open symbols) are shown. The numbers not protected after CHMI \#1 were so low that meaningful associations with protection could not be done. However, in group 3 the unprotected subjects had the lowest levels of antibodies to PfCSP, both less than 3,500 (Figure 4A and Supplemental Table 4). Since there were only 2 unprotected subjects, meaningful statistical analysis could not be done. Antibodies diminished between the last dose and the 24-week CHMI. The geometric mean (GM) half-life of PfCSP antibodies in groups 1 and 2 was 17.81 weeks ( $n=21$, range 9.61 to 61.76 weeks), and in group 3 was 19.93 weeks ( $n=14$, range 7.78 to 180.88 weeks). There was no significant association between antibodies by any assay (ELISA, aIFA, or ISI) prior to CHMI \#2 and protection.

Approximately $77 \%$ of the antibodies to the rPfCSP in the ELISA were directed against the NANP repeat region of the PfCSP (Supplemental Table 5). By aIFA against PfSPZ, median IgG antibodies were 11.2-fold higher than median IgM antibodies (Supplemental Figure 2). For all 33 subjects in groups 1-3 who underwent CHMI 3 weeks after last dose of vaccine, median IgG antibodies to NF54 PfSPZ were 1.7fold higher than to 7G8 PfSPZ by aIFA in sera taken 2 weeks after the last dose of vaccine $(2,668$ arbitrary 
Table 2. Results of CHMI done 3 weeks and 24 weeks after the last dose of PfSPZ Vaccine for the analysis population

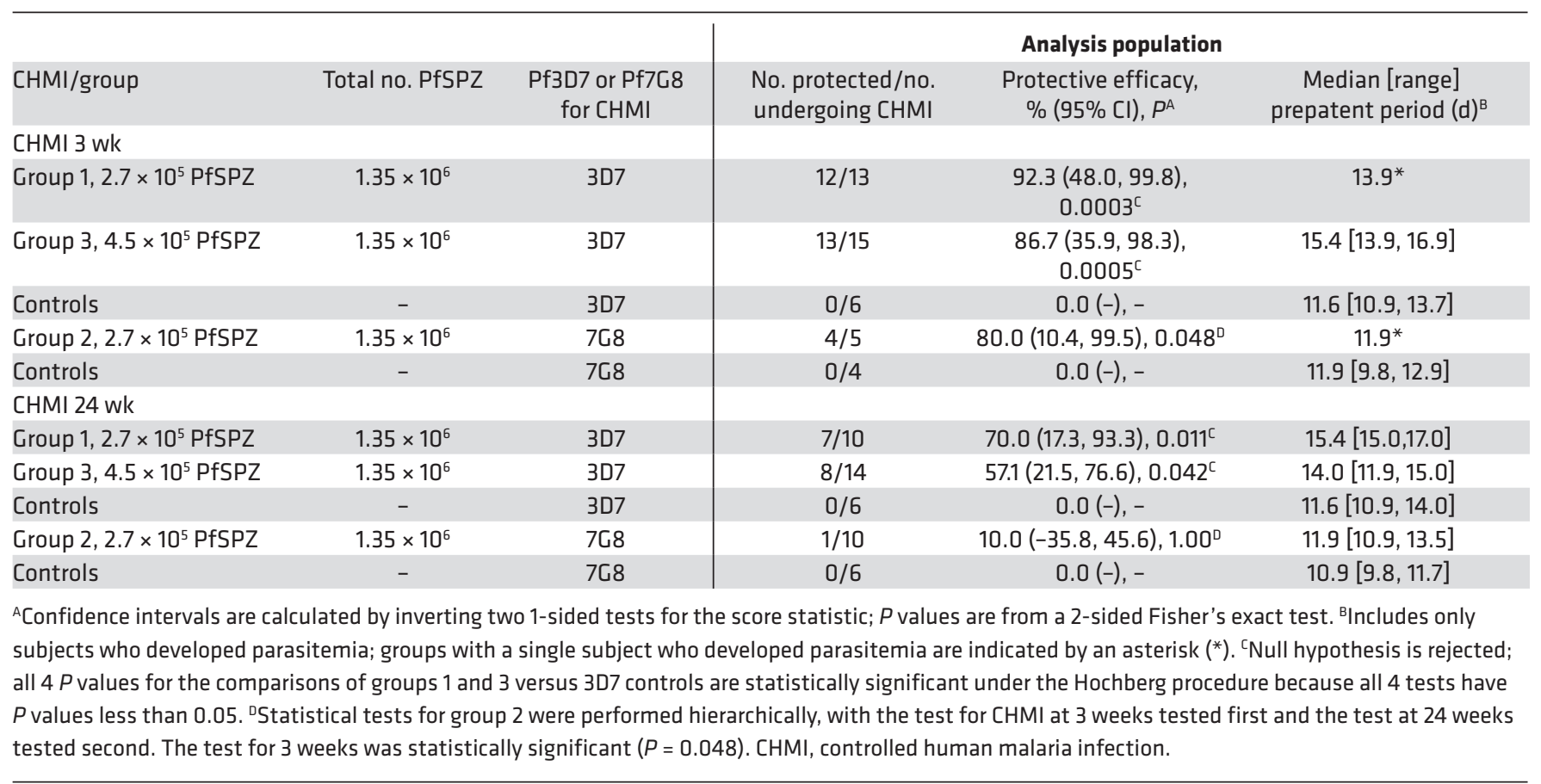

fluorescence units [AFU] $2 \times 10^{5}$ for NF54 [range 0 to 19,106 ] vs. 1,562 AFU $2 \times 10^{5}$ for 7 G8 [range 21 to 10,304], $P=0.016$, Wilcoxon rank-sum test, 2-tailed).

Other antigens. Two weeks after the fifth dose in groups 1 and 2 and after the third dose in group 3, there were no antibodies to PfMSP1, PfEBA175, PfCelTOS, PfEXP1, or PfLSA1 (Supplemental Table 6). At the same time points, there were antibodies to, (a) PfMSP5 in 9 of $26(35 \%)$ subjects in groups 1 and 2, and 12 of $15(80 \%)$ in group 3 ( $P=0.009$, Fisher's exact test, 2-tailed); (b) PfAMA1 in 12 of $26(46 \%)$ subjects in groups 1 and 2, and 1 of $15(6.7 \%)$ in group $3(P=0.014)$; and (c) PfSSP2/TRAP in 6 of $26(23 \%)$ subjects in groups 1 and 2 , and 2 of $15(13 \%)$ in group $3(P=0.687)$. Because of the low numbers of unprotected vaccinees, we could not establish whether any antibody responses were associated with protection.

\section{Cellular immune responses}

Cellular immune responses to stimulation with irradiated PfSPZ were assessed in PBMCs from fresh blood by FluoroSpot 2 (IL-2 and IFN- $\gamma$ ) 2 weeks after the first dose of PfSPZ Vaccine, 2 weeks after the last dose of vaccine (1 week before CHMI\#1), and 23 weeks after the last dose of vaccine (1 week before CHMI \#2). Responses were in general 1.5-3 times higher after the first dose than 2 weeks after the last dose of vaccine, but there were no significant differences between these time points. There were essentially no measurable

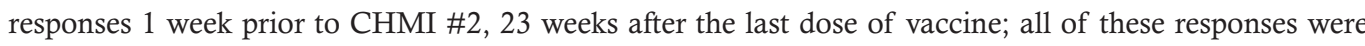
significantly lower than prior to CHMI \#1 (Kruskal-Wallis test, all $P<0.0002$ ). There was no clear association between these cellular immune responses to PfSPZ or Pf-derived synthetic peptides and protection (Figure 4, D-F, Supplemental Figure 3, and Supplemental Table 4).

Ease of administration, tolerability, and safety

DVI was rapid, required a single stick $96 \%$ (182 of 190) of the time, and was well tolerated. Subjects reported no pain $73 \%$ of the time and mild pain $24 \%$ of the time (Supplemental Table 7 ).

Details of adverse events (AEs) are presented in Tables 3 and 4 and Supplemental Tables 8-10. Briefly, of the 66 solicited AEs reported within 7 days of immunization considered related, 61 (92\%) were grade 1 and $5(8 \%)$ grade 2 . No subject experienced a grade 3 or 4 solicited AE. All unsolicited AEs reported within 7 days of immunization were grade 1 . The incidence of AEs was not increased in subjects who received $4.5 \times 10^{5} \mathrm{PfSPZ}$ as compared to $2.7 \times 10^{5} \mathrm{PfSPZ}$ (Supplemental Table 8). The incidence of AEs 
A

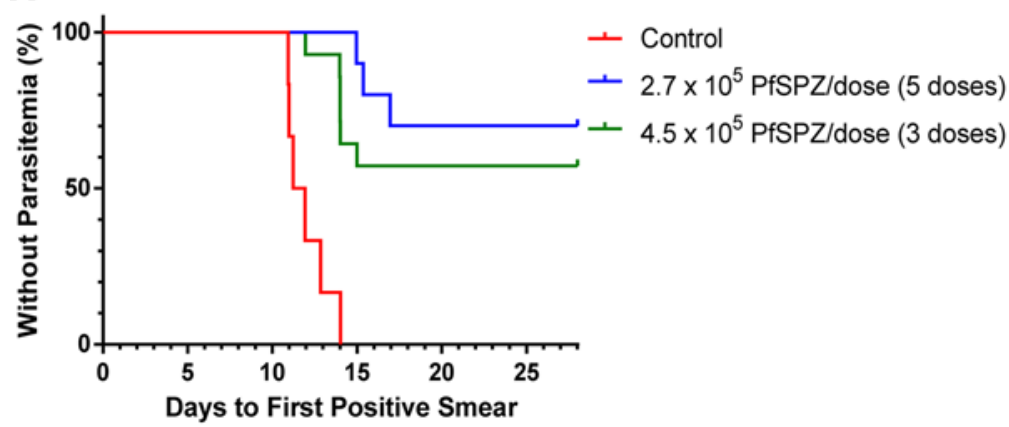

B

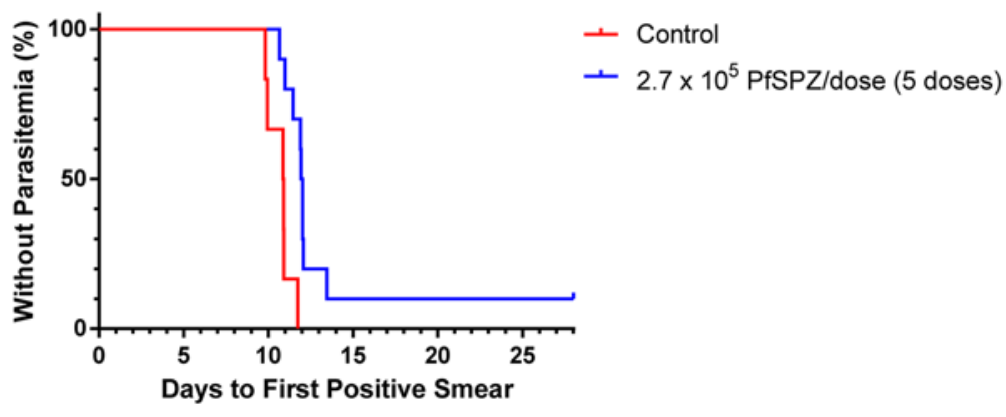

Figure 3. Results of CHMI done 24 weeks after the last dose of PfSPZ Vaccine. Survival curves in volunteers undergoing CHMI 24 weeks after last of 5 doses of $2.7 \times 10^{5} \mathrm{PfSPZ}$ ( $\mathbf{A}$ and B) or 3 doses of $4.5 \times 10^{5} \mathrm{PfSPZ}$ (A). Homologous CHMI with Pf $3 D 7$ parasites was analyzed in two immunized groups (group 1 [2.7 $\left.\times 10^{5} \mathrm{PfSPZ}\right]: n=10$; group 3 [4.5 $\left.\left.\times 10^{5} \mathrm{PfSPZ}\right]: n=14\right)$ and one control group $(n=6)(\mathbf{A})$. Heterologous CHMI with Pf 7G8 parasites was analyzed in one immunized group (group 2 [2.7 $\times$ $10^{5}$ PfSPZ]: $n=10$ ) and one control group $(n=6)(B)$. CHMI, controlled human malaria infection; $\mathrm{Pf}=$ Plasmodium falciparum.

did not increase as subjects received additional doses of the vaccine (Supplemental Tables 9 and 10). 31 of the 45 immunized subjects ( $75 \%$ ) had at least one abnormal laboratory value within 7 days of an immunization, but there was no association with dosing schedule, size, or sequence (Table 4). Details on laboratory abnormalities are provided in Supplemental Table 11.

\section{Discussion}

An important first step in our development program is to optimize the immunization regimen for non-immune individuals who travel to malarious areas. Their risk was underscored by the recent deaths from malaria of two airline flight attendants after exposure of as little as 1

day $(10,11)$. For this population we are aiming for a vaccine that is easy to administer, extremely well tolerated, and safe and at a minimum prevents all $\mathrm{Pf}$ erythrocytic-stage parasitemia in at least $80 \%$ of recipients for 3 months (short-term travel) and ideally 6 months or longer.

Our trial designs have been based on the hypothesis (Figure 5) that increasing the number of PfSPZ/ dose and altering intervals between doses will lead to moving from no or minimal protection to high-level $(\geq 80 \%$ ) short-term protection against homologous parasites (threshold 1); high-level short-term protection against heterologous parasites (threshold 2); high-level long-term protection against homologous parasites (threshold 3); and eventually high-level long-term protection against heterologous parasites (threshold 4). In the first successful trial we reached threshold 1, but did not assess for thresholds 2 and 3 (4).

In this trial, short-term protection against homologous and heterologous $\mathrm{CHMI}$ was $\geq 80 \%$. At 3 weeks after the last dose of vaccine, $92 \%$ of 13 subjects immunized with a total of $1.35 \times 10^{6} \mathrm{PfSPZ}$ in a 5 -dose regimen, and $87 \%$ of 15 subjects immunized with $1.35 \times 10^{6} \mathrm{PfSPZ}$ in a 3-dose regimen were protected against homologous CHMI, reaching threshold 1. Furthermore, this trial gave us an indication that there was short-term protection (threshold 2) against heterologous CHMI. All 4 controls developed parasitemia, and 4 of the 5 vaccinees did not develop parasitemia (protective efficacy of $80 \%, 95 \%$ CI: 10.4, 99.5).

At 24 weeks after the last dose of vaccine, $70 \%$ of 10 subjects immunized with the 5-dose regimen and $57 \%$ of 14 subjects immunized with the 3-dose regimen were protected against homologous CHMI, nearly reaching the $80 \%$ level required to achieve threshold 3 . Furthermore, recent data indicate that if protected at 6 months, vaccinees will be protected at $\sim 14$ months (7).

However, only $10 \%$ of 10 subjects immunized with the 5-dose regimen were protected against heterologous CHMI. Nonetheless, there was a significant difference in time to onset of parasitemia in immunized versus control subjects (Figure 3), suggesting vaccine-induced immunity had prevented infection of or eliminated most liver-stage parasites $(12,13)$. Based on our hypothesis (Figure 5), in the next trial we will immunize with higher doses of PfSPZ in 3-dose regimens. If this is not as effective as expected, we may move to a vaccine containing a mixture of strains of Pf.

PASPZ Vaccine was well tolerated and safe. A study done in Mali with the same dosage regimen with a blinded control group had the same incidence rates of AEs in vaccine and normal saline placebo recipients (Mahamadou S. Sissoko, Malaria Research and Training Center, Mali-NIAID ICER, University of Science, Techniques and Technologies of Bamako, Mali; and Sara A. Healy, Laboratory of Malaria Immunology and Vaccinology, National Institute of Allergy and Infectious Diseases, NIH; personal communication). In our 
Table 3. Solicited and unsolicited adverse events ${ }^{\mathrm{A}}$

\begin{tabular}{|c|c|c|c|c|}
\hline & \multicolumn{2}{|c|}{ Groups 1 and 2 ( 5 doses of $2.7 \times 10^{5}$ PfSPZ), $n=30$} & \multicolumn{2}{|c|}{ Group 3 ( 3 doses of $4.5 \times 10^{5} \mathrm{PfSPZ}$ ), $n=15$} \\
\hline & $n(\%)$ with grade $1 \mathrm{AE}$ & $n(\%)$ with grade $2 \mathrm{AE}$ & $n(\%)$ with grade $1 \mathrm{AE}$ & $n(\%)$ with grade $2 \mathrm{AE}$ \\
\hline $\begin{array}{l}\text { Solicited AE at any } \\
\text { immunization }\end{array}$ & $14 / 30(47)$ & $2 / 30(7)$ & $5 / 15(33)$ & $3 / 15(20)$ \\
\hline $\begin{array}{l}\text { Solicited: local reaction at } \\
\text { injection site }\end{array}$ & $4(13)$ & 0 & $2(13)$ & 0 \\
\hline Pain & $2(7)$ & 0 & $2(13)$ & 0 \\
\hline Induration & $1(3)$ & 0 & 0 & 0 \\
\hline Swelling & 0 & 0 & $1(7)$ & 0 \\
\hline Solicited: systemic reaction & $11(37)$ & $2(7)$ & $5(33)$ & $3(20)$ \\
\hline Headache & $10(33)$ & 0 & $5(33)$ & $2(13)$ \\
\hline Fatigue & $4(13)$ & 0 & $2(13)$ & $1(7)$ \\
\hline Arthralgia & $1(3)$ & $2(7)$ & 0 & 0 \\
\hline Diarrhea & $1(3)$ & 0 & 0 & 0 \\
\hline $\begin{array}{l}\text { Unsolicited AE at any } \\
\text { immunization }\end{array}$ & $14 / 30(47)$ & $0 / 30$ & $4 / 15(27)$ & $0 / 15$ \\
\hline Injection site hemorrhage & $4(13)$ & 0 & $1(7)$ & 0 \\
\hline Cough & $3(10)$ & 0 & 0 & 0 \\
\hline Pyrexia & $2(7)$ & 0 & $1(7)$ & 0 \\
\hline Rhinorrhea & $2(7)$ & 0 & $1(7)$ & 0 \\
\hline Injection site bruising & $2(7)$ & 0 & 0 & 0 \\
\hline Abdominal pain & 0 & 0 & $1(7)$ & 0 \\
\hline Dizziness & $1(3)$ & 0 & 0 & 0 \\
\hline
\end{tabular}

study injection by DVI took only 10-15 seconds after the injection site was prepared and caused no pain in $73 \%$ of immunizations administered, mild pain in $24 \%$, and moderate pain in 3\% (Supplemental Table 7). Administration of PfSPZ Vaccine by DVI appears to be much better tolerated than vaccines administered by traditional routes, likely because all vaccine leaves the site of administration rapidly and there are no inflammatory components in the vaccine. In traditional administration, the most discomfort is experienced when the vaccine is injected and for several hours afterward. Most subjects who received PfSPZ Vaccine by DVI did not sense injection. This will be an advantage in mass vaccine administration campaigns, and in acceptability by infants, children, and travelers.

Through the mid-1980s it was thought that antibodies to PfSPZ, particularly to the PfCSP, that blocked sporozoite invasion of hepatocytes were responsible for irradiated SPZ-induced protection $(14,15)$. However, when it was shown that treatment of fully immune mice $(6,16-18)$ and non-human primates (19) with anti-CD8 antibodies eliminated the protective immunity, it was hypothesized that cellular immune responses against the developing liver stages were responsible for protection $(16,17,20-23)$. We could not use statistics to assess correlations between antibodies and protection for CHMI \#1, and there was no significant correlation between antibodies and protection for CHMI \#2 (Figure 4). This is consistent with our 
A

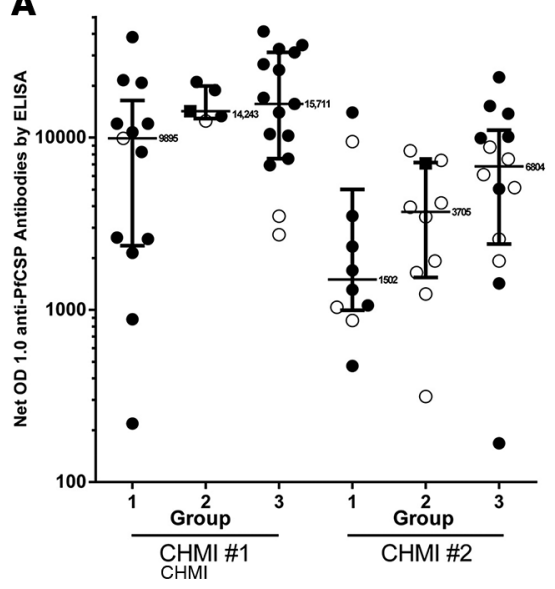

B

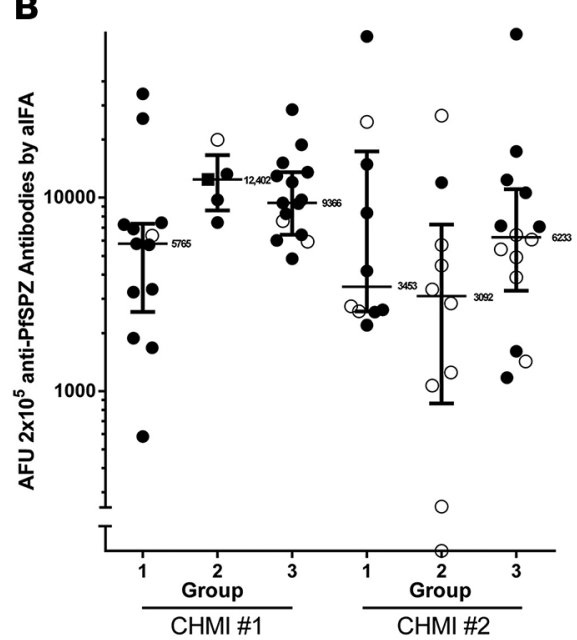

C

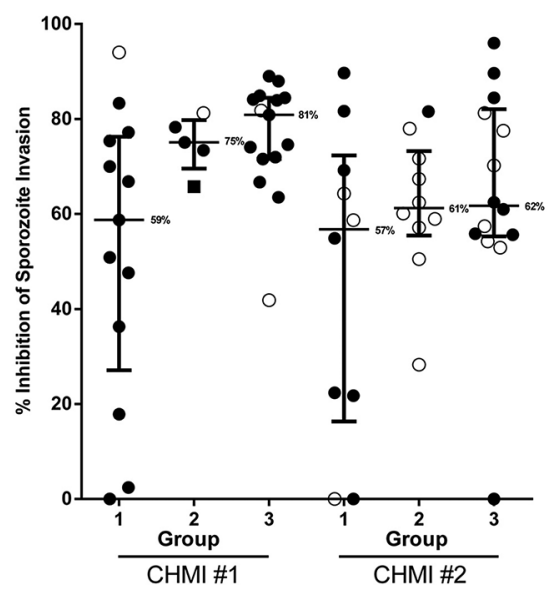

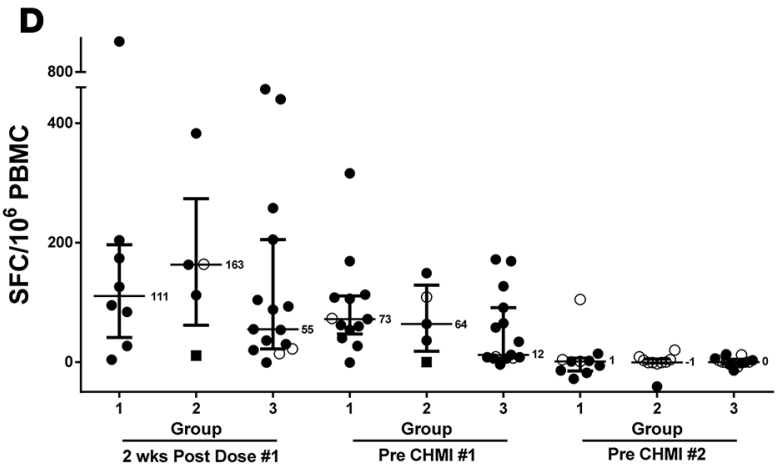

E
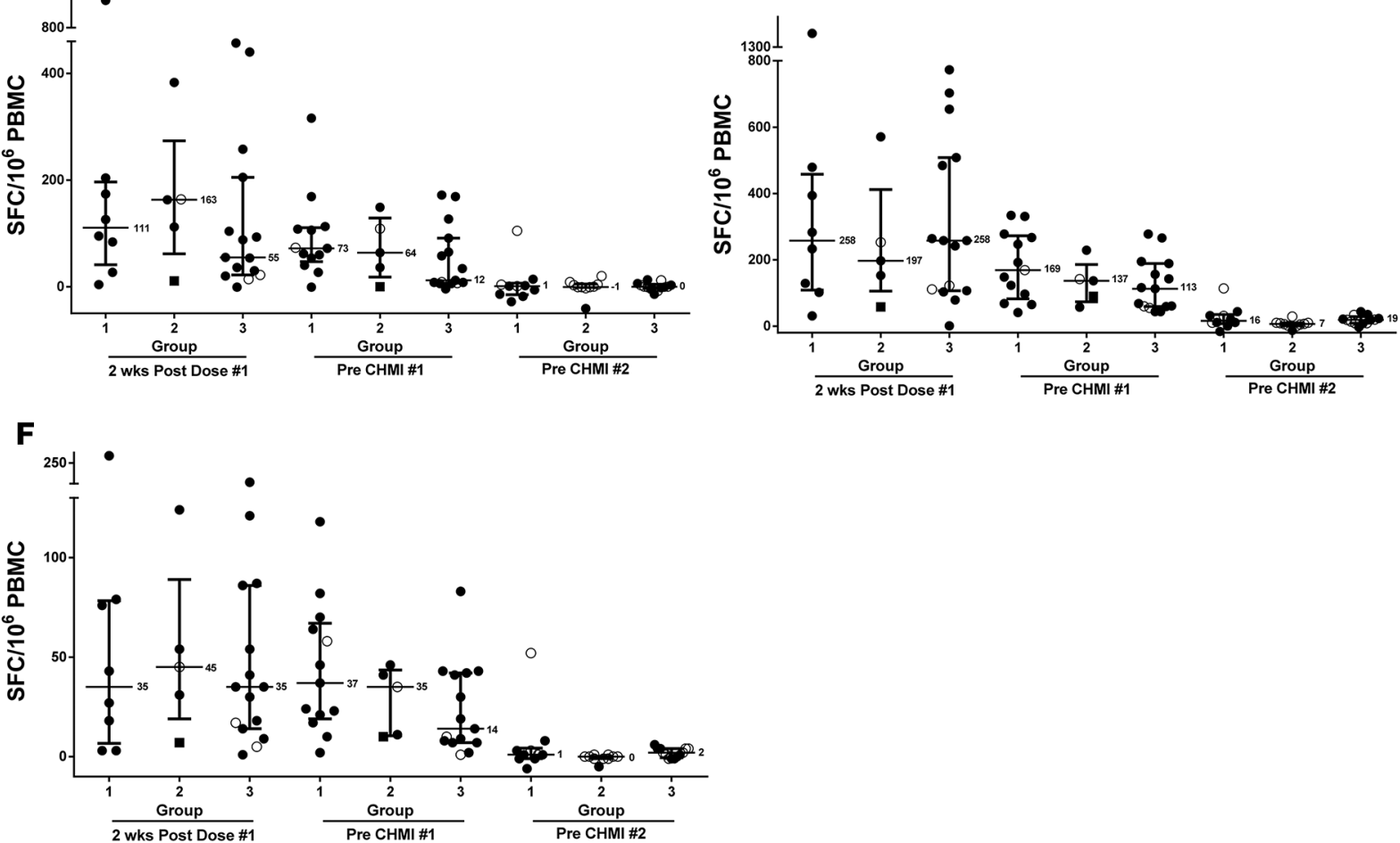

Figure 4. Antibody and T cell responses in all immunized participants. For all assays, protected subjects are shown as filled (black) circles and unprotected subjects as open circles. For each of the 3 immunization groups, the interquartile ranges and the median values of responses of subjects in each group are shown. Assessment of antibodies (A-C) was performed in sera from subjects before immunization and 2 and 23 weeks after the last dose of PfSPZ Vaccine, time points that were 1 week before $\mathrm{CHMI} \# 1$ and $\mathrm{CHMI} \# 2$, respectively. Number of samples assessed in $\mathbf{A}-\mathbf{C}$ for $\mathrm{CHMI} \# 1$ : group 1 ( $n=$ $13)$, group $2(n=5)$, group $3(n=15)$; CHMI \#2: group $1(n=10)$, group $2(n=10)$, group $3(n=14)$. (A) Antibodies to PfCSP by ELISA are reported as net OD 1.0 (the difference in OD 1.0 between post- and pre-immunization sera). (B) Antibodies to PfSPZ by alFA are reported as the reciprocal serum dilution at which the fluorescence units were $2 \times 10^{5}\left(\mathrm{AFU} 2 \times 10^{5}\right)$. (C) Percent inhibition of PfSPZ invasion is reported as the percent reduction of the numbers of PfSPZ that invaded a human hepatocyte line (HC-04) in the presence of post-immunization as compared with pre-immunization serum from the same volunteer, both at a dilution of 1:5. At both CHMI \#1 and CHMI \#2, all 3 assays correlated with each other (CHMI \#1: PfCSP ELISA vs. alFA, $P<$ $0.001, r^{2}=0.61$; PfCSP ELISA vs. ISI, $P=0.0002, r^{2}=0.30$; alFA vs. ISI, $P=0.004, r^{2}=0.19$. CHMI \#2: PfCSP ELISA vs. alFA, $P<0.0001, r^{2}=0.54 ;$ PfCSP ELISA vs. ISI, $P<0.0001, r^{2}=0.56$; alFA vs. ISI, $\left.P=0.0002, r^{2}=0.34\right)$. Assessment of T cell responses by FlouroSpot assay was performed using PBMCs from subjects prior to immunization, 2 weeks after the first dose of PfSPZ Vaccine, and just prior to CHMI \#1 and CHMI \#2 (D-F). Number of samples assessed in D-F -2 weeks after dose 1: group $1(n=8)$, group $2(n=5)$, group $3(n=15)$; pre-CHMI \#1: group $1(n=13)$, group 2 ( $n=5)$, group 3 ( $n=15)$; pre-CHMI \#2: group $1(n=10)$, group $2(n=10)$, group $3(n=13)$. Results are reported as spot-forming cells (SFCs) per $10^{6}$ PBMCs secreting $(\mathbf{D}-\mathbf{F})$ IFN- $\gamma$ only, (E) IL-2 only, or (F) IFN- $\gamma$ and IL-2. Individual data points have the pre-immunization SFCs/10 $\mathrm{PBMCs}$ subtracted from the post-immunization SFCs $/ 10^{6}$ PBMCs. alFA, automated immunofluorescence assay; ISI, inhibition of sporozoite invasion. 
Table 4. Laboratory abnormalities occurring within 7 days of any immunization

\begin{tabular}{|c|c|c|c|c|}
\hline & \multicolumn{2}{|c|}{$\begin{array}{c}\text { Groups } 1 \text { and } 2 \\
\text { ( } 5 \text { doses of } 2.7 \times 10^{5} \mathrm{PfSPZ} \text { ), } n=30\end{array}$} & \multicolumn{2}{|c|}{$\begin{array}{c}\text { Group } 3 \\
\text { (3 doses of } 4.5 \times 10^{5} \text { PfSPZ), } n=15\end{array}$} \\
\hline & $\begin{array}{c}\text { n (\%) with grade } 1 \\
\text { abnormal lab }\end{array}$ & $\begin{array}{c}n(\%) \text { with grade } 2 \\
\text { abnormal lab }\end{array}$ & $\begin{array}{c}n(\%) \text { with grade } 1 \\
\text { abnormal lab }\end{array}$ & $\begin{array}{c}\boldsymbol{n}(\%) \text { with grade } 2 \\
\text { abnormal lab }\end{array}$ \\
\hline Any laboratory abnormality & $15 / 30(50)$ & $6 / 30(20)$ & $5 / 15(33)$ & 4/15 (27) \\
\hline Decreased hemoglobin & $5(17)$ & $2(7)$ & $4(27)$ & 0 \\
\hline Decreased WBC & $4(13)$ & $1(3)$ & $3(20)$ & $1(7)$ \\
\hline Elevated AST & $9(30)$ & $2(7)$ & 0 & 0 \\
\hline Elevated ALT & $6(20)$ & $1(3)$ & $2(13)$ & 0 \\
\hline Elevated creatinine & $5(17)$ & 0 & $2(13)$ & 0 \\
\hline Elevated BUN & $2(7)$ & 0 & $1(7)$ & 0 \\
\hline Elevated total bilirubin & $2(7)$ & 0 & $1(7)$ & 0 \\
\hline
\end{tabular}

hypothesis that antibodies can reduce the numbers of PfSPZ that reach or successfully invade hepatocytes, but cellular immune responses directed against perhaps hundreds of antigens expressed in developing liver stages are required for the sterile protective immunity induced by PfSPZ Vaccine $(4,6)$.

The cellular immune responses delineated by the FluoroSpot assays peaked after the first dose, were lower after the last dose, and essentially absent at the time of CHMI \#2. There was no significant association with protection. This may be because, as in non-human primates, the majority of PfSPZ-specific $\mathrm{CD}^{+}$cells are in the liver, where they can attack the Pf-infected hepatocyte, not in the blood, where we can measure them (6). Given that there has been association between $\mathrm{T}$ cell responses and protection as assessed by flow cytometry $(4,7)$, we are proceeding with such an analysis. The low levels of antibodies (Supplemental Table 6) and T cell responses (Supplemental Figure 3) against well-known antigens suggest that as-yet-undefined antigens may be critically important targets of this strong protective immunity, and these may be revealed by flow cytometry studies.

We believe CHMI with heterologous Pf strains will be adequate to predict protective efficacy in the field against heterogeneous Pf populations. This is supported by our study in malaria-exposed Malian adults immunized with the same regimen as groups 1 and 2 in this study, which conferred $28.5 \%$ sterile protection against highly heterogeneous naturally transmitted Pf for up to 6 months (M.S. Sissoko and S.A. Healy, personal communication) - several fold better protection than the 10\% protective efficacy against Pf7G8 at 24 weeks in the present study. Recent evidence has suggested that the RTS,S/AS01 vaccine induces strain-specific protection in the field (24). Only homologous CHMI has been used in the development and testing of RTS,S/AS01. It is likely that heterologous CHMI would have identified this deficiency early on. It is for this reason we have conducted the first heterologous CHMI, and will continue to use heterologous CHMI in the development of PfSPZ Vaccine.

Whole genome sequencing revealed that the genetic distance between the vaccine strain (Pf3D7, a proxy for its parent strain PfNF54) and Pf7G8, the two strains used for CHMI, was substantial (22,056 SNPs). Furthermore, a targeted analysis of 13 known pre-erythrocytic antigens revealed that they all contain non-synonymous SNPs between Pf3D7 and Pf7G8. Thus, we conclude that Pf7G8 is an appropriate parasite for heterologous CHMI.

By increasing the dose (Figure 5), we anticipate we will increase the magnitude of protective immune responses against all target antigens, and will expand the immune responses to be direct against subdominant protective and conserved epitopes. They are conserved because there is no immune pressure against these subdominant epitopes, and this should allow for protection against all strains of Pf. We anticipate finalization of 


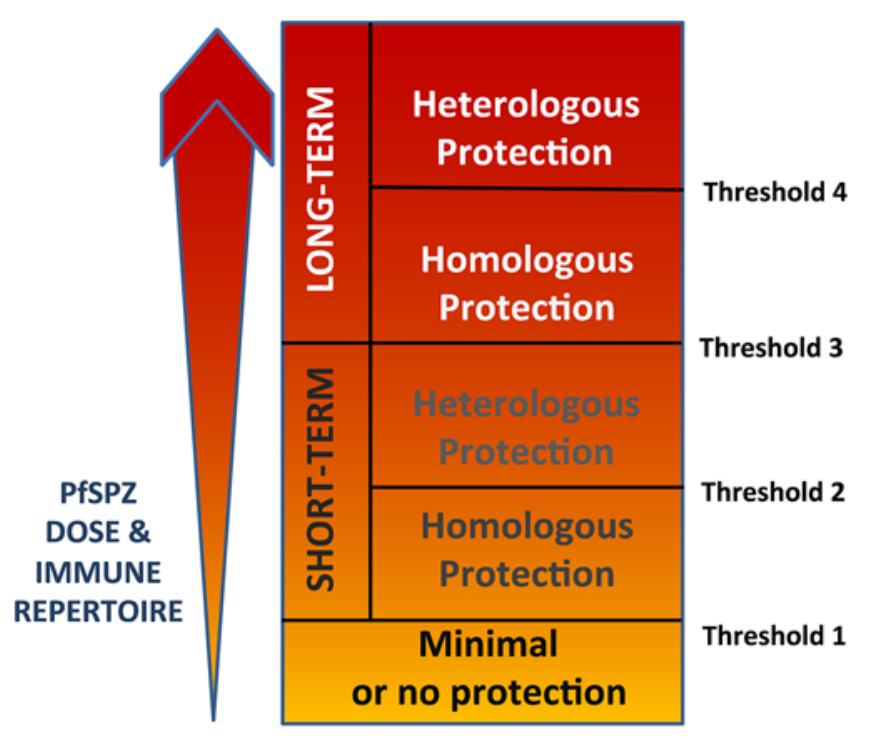

Technical Requirements for Pharmaceuticals for Human Use.

The NMRC, WRAIR, and Sanaria (sponsor) collaborated on design. There was an independent safety monitoring committee (SMC).

\section{Study subjects}

Malaria-naive, 18- to 45-year-olds were enrolled during 2014-2015 after providing informed consent. Subjects were excluded if they had a history of malaria infection, travel to a malaria endemic region within 6 months of first immunization, or long-term residence ( $>5$ years) in an area known to have transmission of $P$. falciparum. Subjects were also excluded if they had previously participated in a malaria vaccine trial. All subjects underwent a screening evaluation consisting of a medical history, physical examination, electrocardiogram, complete blood count, clinical biochemistries, urinalysis, sickle cell testing, and serological studies for previous exposure to or infection with HIV, hepatitis B, and hepatitis C. Subjects were excluded if they had hepatic, renal, or autoimmune diseases, allergy to any study component, splenectomy, or evidence of increased cardiovascular disease risk (defined as $>10 \%$, 5-year risk) as determined by the method of Gaziano (25). All females had urine pregnancy test at screening, immediately before each immunization and before CHMI; they were to be excluded from further immunization or CHMI if this was positive. All female subjects agreed to use effective means of birth control for the duration of the trial.

\section{PfSPZ Vaccine}

Sanaria PfSPZ Vaccine is a live attenuated whole parasite vaccine (26) stabilized in liquid nitrogen vapor phase (LNVP), and manufactured and characterized (Supplemental Table 12) as described previously (46). Vaccine in $0.5 \mathrm{ml}$ was rapidly (seconds) injected in an arm vein by DVI through a 25 -gauge needle.

\section{Study design}

The study was open label and designed to assess the tolerability, safety, immunogenicity, and protective efficacy of DVI-administered PfSPZ Vaccine in 3 groups of 15 immunized subjects each. Thirty subjects were to receive 5 doses of $2.7 \times 10^{5} \mathrm{PfSPZ} /$ dose at weeks $0,4,8,12$, and 20 and then were to be randomized equally to group 1 (homologous CHMIs) or group 2 (heterologous CHMIs). Fifteen subjects in group 3 were to receive 3 doses of $4.5 \times 10^{5} \mathrm{PfSPZ} /$ dose at weeks 0,8 , and 16 , followed by homologous CHMIs.

Tolerability of DVI was assessed immediately after injection by the subjects using a pain scale (none, mild, moderate, or severe). Subjects were followed up in the clinic on days 2, 7, and 14 after each immunization. Solicited and unsolicited AEs were monitored for 7 and 28 days after each immunization, respectively. Safety laboratories were assessed 2 and 7 days after each immunization. A TBS was assessed $14 \pm 2$ days after the first immunization to rule out breakthrough infection. See supplemental material for details of $\mathrm{AE}$ recording and grading. 
CHMI was conducted 3 and 24 weeks after the final immunization. Groups 1 and 3 underwent CHMI by bites of 5 Anopheles stephensi mosquitoes infected with Pf3D7 parasites (27), which are a clone of the NF54 parasites in the vaccine (presumed African origin) (homologous CHMI). Group 2 underwent CHMI by bites of $5 \mathrm{~A}$. stephensi infected with Pf7G8 parasites (South American) (heterologous CHMI) (28). As is standard for malaria vaccine trials in malaria-naive subjects with CHMI, for each CHMI there were non-immunized infectivity controls. From day 7 to day 18 after exposure to the bites of PfSPZ-infected mosquitoes, subjects spent evenings and nights in a hotel. AEs and TBSs were assessed at least daily from day 7 to day 18 after CHMI, and then again on days 20, 22, 25, and 28 after initiation of CHMI or until detection of parasitemia as previously described (13). qPCR was done retrospectively (see supplemental materials for specifics) $(29,30)$. Clinical laboratory tests were also monitored. Upon detection of parasites, subjects were treated with a 3-day course of atovaquone/ proguanil (Malarone).

\section{Assessment of immunological responses}

Antibody assays. Sera were assessed for antibodies to selected Pf proteins by ELISA, and to Pf sporozoites by aIFA. Functional activity of sera was assessed by the ISI assay. See supplemental materials for specifics.

Cellular immunology assays. PBMCs were assessed for activity against PfSPZ and Pf peptides by FluoroSpot assay (Mabtech). See supplemental material for specifics.

\section{Assessment of genetic differences between $P$. falciparum isolates}

SNP calling utilizing previously generated whole-genome short-read sequencing data was used to estimate genetic distance between the vaccine strain PfNF54 and Pf7G8, as well as between these and 19 clinical isolates from Africa (Supplemental Table 1 and Supplemental Figure 1), with details described in the supplemental material. Given the negligible difference (55 detectable SNPs) between the Pf3D7 clone and its parent, PfNF54, 3D7 (27) was used as a proxy for NF54 (31).

\section{Statistics}

Sample sizes for the groups were selected based on comparison of the number of immunized subjects per group versus 6 infectivity controls for each CHMI. Accounting for a dropout rate of immunized subjects of $20 \%$ (from 15 to 12 subjects), a sample size of 6 in the control group and 12 in the vaccinated group was selected to be able to show with a power of $80 \%$ without a continuity correction that if 5 of 6 become infected in the control group and 2 of 12 become infected in the immunized group, the proportion of subjects infected in the control group would be significantly different from the proportion of subjects infected in the immunized group ( $\alpha<0.05,2$-sided Fisher's exact test).

Immunological analyses, differences in responses were analyzed by nonparametric tests.

Protective efficacy was calculated as 1 - (relative risk of parasitemia infection). Exact CIs for protective efficacy were calculated by inverting two 1-sided tests for the score statistic in StatXact 9 (Cytel 2010, Cytel Studio 9). Statistical comparison tests of protective efficacy were performed using 2-sided Fisher's exact tests comparing vaccinated subjects with controls within similar CHMI strains. The Hochberg procedure was applied to the comparisons of the 3D7 strain CHMIs (group 1 versus control and group 3 versus control for both CHMIs), for a total of 4 comparisons to preserve the overall type I error rate at 0.05 (32). A hierarchical testing procedure was applied to the comparison for the 7G8 strain CHMIs (group 2 versus controls). Because a priori we believed that protection was more likely at 3 weeks than at 24 (see Discussion and Figure 5), we planned to perform the 3-week comparison first at a type I error rate of 0.05 and, only if that was significant, use 0.05 for the 24 -week CHMI. Analyses were performed on two populations: (a) the analysis population and (b) the challenged population. The analysis population consisted of fully immunized subjects who participated in the respective CHMI. The text of this article reports results for the analysis population. The challenged population consisted of all subjects who received the respective CHMI, regardless of the number of vaccinations received or compliance status. Results for the challenged population are shown in Supplemental Table 3.

\section{Study approval}

The IRB of WRAIR approved the protocol in compliance with applicable federal regulations. Written informed consent was obtained from all subjects prior to inclusion in the study. 


\section{Author contributions}

JEE, KMP, BKLS, S Reyes, EV, TLR, and SLH contributed to the study design. Protocol development and conduct of the clinical trial were performed by JEE, KMP, MS, A Singer, A Stafford, RCR, RN, DT, S Cicatelli, SD, LG, ES, PT, S Reyes, JEM, CV, S Remich, BH, and JR. AJR, S Chakravarty, AGE, TL, PFB, AM, ERJ, NKC, ML, YA, BKLS, and SLH were involved with vaccine manufacture. Regulatory support was provided by TM and AG. MS, S Chakravarty, JCS, HG, KM, NKC, ML, EA, AB, KH, MB, JH, and BKLS performed the assays. EA, AB, and MS carried out the thick smear microscopy. JEE, TLR, AJR, JC, AT, DP, and SLH conducted the data analysis. JEE, TLR, AJR, and SLH wrote the manuscript, and MS, S Chakravarty, JC, S Reyes, MC, YA, and AT assisted with final manuscript preparation.

\section{Acknowledgments}

We thank the volunteers for their participation and the PfSPZ Vaccine Development Teams (see supplemental material) for their incredible efforts. The trial was supported primarily by Department of Defense Award/Contract W81XWH1420011; this included production and characterization of the vaccine. Additional funds were provided by the Naval Medical Research Center's Advanced Medical Development Program and the Military Infectious Diseases Research Program. At the NMRC, work was funded by Work Unit Number A1217. JCS and KM were supported by grant U19AI10820 from the National Institute of Allergy and Infectious Diseases. All of the data reported in the manuscript are tabulated in the main text and in the supporting online material. A number of patents on PASPZ have been issued, allowed, or filed in the USA and internationally.

Address correspondence to: Stephen L. Hoffman, Sanaria Inc., 9800 Medical Center Drive, Suite A209, Rockville, Maryland 20805, USA. Phone: 240.403.2701; E-mail: slhoffman@sanaria.com.

KMP's present address is: Center for Global Health and Translational Sciences, Upstate Medical University, New York, USA. AS's present address is: Virginia Cancer Specialists, Arlington, Virginia, USA. RCR's present address is: U.S. Army's Health Clinic-Schofield Barracks, Schofield Barracks, Hawaii, USA. RN's present address is: Clinical Research Management, Frederick, Maryland, USA. DP's present address is: University of Virginia, Charlottesville, Virginia, USA. SD's present address is: Armed Forces Research Institute of Medical Science, Bangkok, Thailand. KH's present address is: U.S. Army Medical Directorate-Armed Forces Research Institute of Medical Services, Bangkok, Thailand. CV's present address is: Bio Clinica Inc., Newark, California, USA. MC's present address is: USS Ashland LSD 48. BH's present address is: Navy Experimental Diving Unit Medical Department, Panama City, Florida, USA. JR's present address is: Department of Clinical Research, U.S. Army Medical Research Institute of Infectious Diseases, Frederick, Maryland, USA.

1. World Malaria Report 2015. World Health Organization. http://www.who.int/malaria/publications/world-malaria-report-2015/report/en/. Accessed November 28, 2016.

2. Hoffman SL, et al. Protection of humans against malaria by immunization with radiation-attenuated Plasmodium falciparum sporozoites. J Infect Dis. 2002;185(8):1155-1164.

3. Roestenberg M, et al. Protection against a malaria challenge by sporozoite inoculation. N Engl J Med. 2009;361(5):468-477.

4. Seder RA, et al. Protection against malaria by intravenous immunization with a nonreplicating sporozoite vaccine. Science. 2013;341(6152):1359-1365.

5. Hoffman SL, et al. Development of a metabolically active, non-replicating sporozoite vaccine to prevent Plasmodium falciparum malaria. Hum Vaccin. 2010;6(1):97-106.

6. Epstein JE, et al. Live attenuated malaria vaccine designed to protect through hepatic CD8+ T cell immunity. Science. 2011;334(6055):475-480.

7. Ishizuka AS, et al. Protection against malaria at 1 year and immune correlates following PfSPZ vaccination. Nat Med. 2016;22(6):614-623.

8. Malaria Vaccine Funders Group. Malaria Vaccine Technology Roadmap. World Health Organization. http://www.who.int/immunization/topics/malaria/vaccine_roadmap/TRM_update_nov13.pdf?ua=1. Accessed November 28, 2016.

9. Egan JE, et al. Humoral immune responses in volunteers immunized with irradiated Plasmodium falciparum sporozoites. Am $J$ Trop Med Hyg. 1993;49(2):166-173.

10. Steward treated for malaria in Turkey's west, days after stewardess dies of same disease. Hurriyet Daily News. http://www. hurriyetdailynews.com/steward-treated-for-malaria-in-turkeys-west-days-after-stewardess-dies-of-same-disease-.aspx?page$\mathrm{ID}=238 \& \mathrm{nID}=84450 \&$ NewsCatID=341. Published June 24 2015. Accessed November 28, 2016.

11. Hayward, S. A British Airways flight attendant has died of malaria after a long-haul flight to Africa. Daily Mirror. http:// 
www.mirror.co.uk/news/uk-news/british-airways-stewardess-dies-after-6094210. Published July 18, 2015. Accessed November 28, 2016.

12. Murphy JR, Baqar S, Davis JR, Herrington DA, Clyde DF. Evidence for a 6.5-day minimum exoerythrocytic cycle for Plasmodium falciparum in humans and confirmation that immunization with a synthetic peptide representative of a region of the circumsporozoite protein retards infection. J Clin Microbiol. 1989;27(7):1434-1437.

13. Bejon P, et al. Calculation of liver-to-blood inocula, parasite growth rates, and preerythrocytic vaccine efficacy, from serial quantitative polymerase chain reaction studies of volunteers challenged with malaria sporozoites. J Infect Dis. 2005;191(4):619-626.

14. Nussenzweig V, Nussenzweig RS. Circumsporozoite proteins of malaria parasites. Cell. 1985;42(2):401-403.

15. Hoffman SL, et al. Immunity to malaria and naturally acquired antibodies to the circumsporozoite protein of Plasmodium falciparum. NEngl J Med. 1986;315(10):601-606.

16. Schofield L, Villaquiran J, Ferreira A, Schellekens H, Nussenzweig R, Nussenzweig V. Gamma interferon, CD8+ T cells and antibodies required for immunity to malaria sporozoites. Nature. 1987;330(6149):664-666.

17. Weiss WR, Sedegah M, Beaudoin RL, Miller LH, Good MF. CD8+ T cells (cytotoxic/suppressors) are required for protection in mice immunized with malaria sporozoites. Proc Natl Acad Sci U S A. 1988;85(2):573-576.

18. Doolan DL, Hoffman SL. The complexity of protective immunity against liver-stage malaria. J Immunol. 2000;165(3):14531462 .

19. Weiss WR, Jiang CG. Protective CD8+ T lymphocytes in primates immunized with malaria sporozoites. PLoS One. 2012;7(2):e31247.

20. Kumar S, et al. Cytotoxic T cells specific for the circumsporozoite protein of Plasmodium falciparum. Nature. 1988;334(6179):258-260.

21. Hoffman SL, et al. Sporozoite vaccine induces genetically restricted T cell elimination of malaria from hepatocytes. Science. 1989;244(4908):1078-1081.

22. Romero P, Maryanski JL, Corradin G, Nussenzweig RS, Nussenzweig V, Zavala F. Cloned cytotoxic T cells recognize an epitope in the circumsporozoite protein and protect against malaria. Nature. 1989;341(6240):323-326.

23. Weiss WR, et al. Cytotoxic T cells recognize a peptide from the circumsporozoite protein on malaria-infected hepatocytes. $J$ Exp Med. 1990;171(3):763-773.

24. Neafsey DE, et al. Genetic Diversity and Protective Efficacy of the RTS,S/AS01 Malaria Vaccine. N Engl J Med. 2015;373(21):2025-2037.

25. Gaziano TA, Young CR, Fitzmaurice G, Atwood S, Gaziano JM. Laboratory-based versus non-laboratory-based method for assessment of cardiovascular disease risk: the NHANES I Follow-up Study cohort. Lancet. 2008;371(9616):923-931.

26. Luke TC, Hoffman SL. Rationale and plans for developing a non-replicating, metabolically active, radiation-attenuated Plasmodium falciparum sporozoite vaccine. J Exp Biol. 2003;206(Pt 21):3803-3808.

27. Walliker D, et al. Genetic analysis of the human malaria parasite Plasmodium falciparum. Science. 1987;236(4809):1661-1666.

28. Burkot TR, Williams JL, Schneider I. Infectivity to mosquitoes of Plasmodium falciparum clones grown in vitro from the same isolate. Trans R Soc Trop Med Hyg. 1984;78(3):339-341.

29. Kamau E, Alemayehu S, Feghali KC, Saunders D, Ockenhouse CF. Multiplex qPCR for detection and absolute quantification of malaria. PLoS One. 2013;8(8):e71539.

30. Kamau E, et al. Sample-ready multiplex qPCR assay for detection of malaria. Malar J. 2014;13:158.

31. Ponnudurai T, Leeuwenberg AD, Meuwissen JH. Chloroquine sensitivity of isolates of Plasmodium falciparum adapted to in vitro culture. Trop Geogr Med. 1981;33(1):50-54

32. Hochberg Y. A sharper Bonferroni procedure for multiple tests of significance. Biometrika. 1988;75(4):800-802. 